\title{
Cardiovascular Risk by Gender in an Italian Pilot Study
}

\author{
Elena Matteucci* and Ottavio Giampietro
}

Department of Internal Medicine, University of Pisa, Italy

\begin{abstract}
Part of the international variation in the gender differences in total mortality remains unexplained. Although a continuous decrease in age-standardised death rates was observed in Italy, the difference between male and female rates became relatively small or even reversed (as the rate for ischaemic heart diseases).

We performed an extensive evaluation of cardiovascular risk factors - demographic and clinical characteristics, biochemical parameters, and oxidative biomarkers - in a sample of healthy subjects (94 women and 75 men) from Central Italy and analysed their relationship with the response to ergometer exercise.

In addition to a proportion of smokers similar to that observed in men, a clustering of peculiar female cardiovascular risk factors emerged. This included: higher platelet counts, low participation in leisure-time aerobic physical activities, hypertensive response to exercise (BMI-dependent, unlike in men), and low fraction of heart rate reserve. Physical inactivity and high serum IL-6 levels were independently predictive of having both chronotropic incompetence and abnormal heart rate recovery.
\end{abstract}

Keywords: Sex, risk factors, ergometer exercise, heart rate reserve, interleukin 6, lifestyle.

\section{INTRODUCTION}

Cardiovascular diseases largely contribute the higher mortality in men. Smoking is an important cause of the international variation in the gender differences in total mortality, yet a great part of this variation remains unexplained [1]. Fluctuations in the gender gap in life expectancy suggest the differences reflect environmental as well as genetic factors [1]. Mortality rates declined for ischaemic heart disease (IHD), stroke, and total cardiovascular diseases between 1970 and 2000 in Western Europe. However, the percentage annual change of the cause-specific mortality rates for the age group 45-74 was negative and significant for IHD, total cardiovascular diseases, and all-cause mortality (not for stroke) in Western European men, whereas only for IHD in women [2]. In Italy a continuous decrease in agestandardised death rates was observed (www.euro.who.int/ eprise/main/WHO/informationSources/Data/20011017_1), but the difference between male and female rates became relatively small or even reversed. Indeed, in 2000 the rate for ischaemic heart diseases was $25 \%$ in women $v s 23 \%$ in men.

In this study, we performed an extensive evaluation of cardiovascular risk factors in a sample of healthy subjects from Central Italy and analysed their relationship with the response to ergometer exercise.

\section{METHODS}

Healthy subjects $(94 \mathrm{~F} / 75 \mathrm{M})$ were recruited from the local community. They were taking no drugs, and had no clinical

\footnotetext{
*Address correspondence to this author at the Dipartimento di Medicina Interna, Via Roma 67, 56126 Pisa, Italy; Tel: 0039050 993246; Fax: 0039 050 553414; E-mail: ematteuc@int.med.unipi.it
}

signs or symptoms of illness. All subjects gave their informed consent and the ethical committee of the hospital approved the study.

The medical examination included standardised personal and family health history, physical examination, blood chemistry analysis and exercise test.

Among the risk factors taken into consideration were: demographic and clinical characteristics (body size, blood pressure, cigarette smoking, lifestyle), biochemical parameters (fasting plasma glucose and insulin, HbA1c, blood cell count, lipids, C-reactive protein, fibrinogen, folate, total homocysteine, electrolytes, interleukin 6 , urinary albumin and electrolyte excretion), and biomarkers of oxidative stress or antioxidant protection (plasma thiols, plasma and erythrocyte malondialdehyde, erythrocyte $\mathrm{Na} / \mathrm{H}$ exchange, erythrocyte velocity of ferricyanide reduction) [3-5]. Erythrocyte $\mathrm{Na} / \mathrm{H}$ exchange (RBC NHE) hyperactivity, once considered marker of essential hypertension, is now ascribed to the direct stimulatory effect of oxidative stress [4]. The erythrocyte velocity of ferricyanide reduction (RBC vfcy) is a plasma membrane oxido-reductase that transfers reducing equivalents from intracellular reductants to extracellular oxidants (such as ferricyanide) and thus enables the cell respond to changes in intra- and extra-cellular redox environments [5].

\section{Data Collection}

Demographic, socio-economic and lifestyle information was obtained by Lifestyle European Prospective Investigation of Cancer and Nutrition questionnaire (EPIC) [6]. Subjects reported the daily number of hours they engaged in housework and the weekly number of hours they engaged in physical activities during leisure and during work. Essential 
hypertension was defined as current use of antihypertensive medication or an office blood pressure $\geq 140 / 90 \mathrm{mmHg}$. The body mass index (BMI) was calculated as $\mathrm{kg} / \mathrm{m}^{2}$; the body surface area (BSA) as $\mathrm{m}^{2}=\mathrm{kg}^{0.425} * \mathrm{~cm}^{0.725} * 0.007184$.

\section{Exercise Testing Procedure}

The examination program included measurements of resting heart rate (HR), systolic (SBP) and diastolic (DBP) blood pressure. A maximum, symptom-limited ECGmonitored exercise test was performed according to the Bruce protocol [7]. The maximal exercise-induced change in SBP and DBP relative to maximum exercise capacity was calculated $(\triangle \mathrm{ExSBP} / \mathrm{W}$ and $\Delta \mathrm{ExDBP} / \mathrm{W})[7]$. A hypertensive response to exercise (HRE) was defined as a peak SBP during exercise $\geq 210 \mathrm{mmHg}$ in men and $\geq 190 \mathrm{mmHg}$ in women [8]. Peak HR achieved, the percentage of age-predicted HR achieved, and the increment in HR from standing rest to peak exercise were measured. The chronotropic response index at peak exercise was calculated as the ratio of heart rate reserve (HRR) used to metabolic reserve used at peak stage [9]. Chronotropic incompetence was defined as a fraction of HRR at peak exercise $<0.8$. An abnormal heart rate recovery was considered to be a decrease of $<22$ beats/min (bpm) at 2 $\min$ in recovery [10].

\section{Laboratory Methods}

Fasting venous blood samples and 24-h urine collections were obtained on a single occasion. Glucose, C-reactive protein (CRP), total/HDL cholesterol, triglycerides, and albumin were measured using a MODULAR ANALYTICS SWA (Roche Diagnostics, Italy). LDL cholesterol was calculated by the Friedewald formula. HbA1c was evaluated by BioRad DIAMAT $^{\mathrm{TM}}$ Analyzer System, plasma fibrinogen using an automated coagulation analyzer (Sysmex, Japan), plasma folate and total homocysteine by AxSYM System and IMX System, respectively (Abbott Diagnostics, Italy), insulin by radioimmunoassay (Medgenix Diagnostics, Belgium). Insulin resistance was estimated by homeostasis model assessment (HOMA ${ }_{\text {IR }}$ ). Serum interleukin 6 (IL-6) was assayed by Quantikine HS (R\&D Systems, UK).

Plasma thiols ( $\mathrm{P} \mathrm{SH}$ ), erythrocyte glutathione (RBC GSH), plasma and erythrocyte membrane malondialdehyde (P and RBC MDA), RBC NHE activity, and RBC vfcy were measured as previously reported $[3-7,11]$.

\section{Statistical Analyses}

The results are expressed as mean $\pm \mathrm{SD}$ or geometric mean. Data not normally distributed were log-transformed before t-tests and ANOVA. The level of significance was set at $\mathrm{P}<0.05$. Student's t-test with Bonferroni corrections, Mann-Whitney U-test, ANOVA and Kruskal-Wallis rank test were used to determine differences between independent groups as appropriate. Comparison of categories was by chisquare test. We estimated the associations by Spearman rank correlation analysis, stepwise regression analysis, multiple linear and logistic regression. A Student-Newman-Keuls post hoc test was used to assess the significance of the difference between specific means.

\section{RESULTS}

\section{Demographic and Clinical Characteristics}

The distribution of age and education was similar for female and male respondents (Table 1). Women had lower body size measures than man. The percentages of neversmokers and smokers were similar between women and men (58\% and $24 \%$ vs $49 \%$ and $24 \%$, respectively). Overall, $76 \%$ of respondents were currently employed; the percent unemployed was higher in women $(35 \%)$ than in man $(24 \%)$. A sedentary professional activity was most common ( $85 \%$ and $68 \%$ of female and male respondents, respectively). Higher proportions of respondents with a manual or heavy manual professional activity were observed in men $(32 \%)$ than in women $(15 \%)$. Almost no man performed household activities, whereas the mean duration of household activities was $2.8 \mathrm{~h} /$ day in women. Time dedicated to physical activity was higher in men because of a high participation in exercise, walking and cycling.

Among women, time spent in housework decreased with increasing educational level: $4 \mathrm{~h} /$ day in primary school, $3.4 \pm 1.1$ in secondary school, $2.3 \pm 1.2$ in high school, $2.0 \pm 1.1$ in graduate $(\mathrm{p}<0.0001)$. Among women (not men), leisure time physical activities correspondingly increased: $0.7 \mathrm{~h} / \mathrm{day}$, $1.1 \pm 0.4,1.5 \pm 0.5$, and $1.5 \pm 0.4$, respectively $(\mathrm{p}<0.01)$. Female (not male) SBP decreased with increasing educational level: $138 \mathrm{mmHg}, 125 \pm 15,114 \pm 18,113 \pm 16$, respectively $(\mathrm{p}<0.01)$. Employment status did not change the distribution of these variables in both groups. School level or employment status made no difference with regard to smoking.

\section{Biochemical Parameters and Biomarkers of Redox Status}

Compared with men, women had lower fasting plasma glucose (FPG), haematocrit, LDL cholesterol, triglycerides, plasma total homocysteine, plasma sodium and potassium, urinary sodium excretion corrected by BSA (Table 2). In contrast, women had significantly higher platelet counts as well as higher levels of HDL cholesterol and plasma folate. HOMA $_{\text {IR }}(2.1 \pm 1.4$ in women, $2.3 \pm 1.3$ in men, $p$ 0.2825) was significantly associated $(|\mathrm{r}| 0.53, \mathrm{p}<0.0001)$ with BMI (coefficient 0.03 , t value 7.64$)$ and $P$ MDA $(0.16,2.28)$.

Current smokers had the highest levels of IL-6 (3.3 $\mathrm{pg} / \mathrm{mL}$ vs 2.1 never smokers and 2.4 ex smokers, $\mathrm{p} 0.0028$ ), CRP (3.3 mg/L vs 2.4 and 2.3, p 0.0258), and RBC MDA $(0.4 \pm 0.14 \mathrm{nmol} / \mathrm{L}$ vs $0.33 \pm 0.13$ and $0.35 \pm 0.14, \mathrm{p} 0.0199)$. Plasma folate and total homocysteine concentrations did not differ when subjects were divided according to cigarette use.

Concerning redox biomarkers, women differed from men only in the higher RBC GSH $(0.81 \pm 0.13 \mathrm{mg} / \mathrm{mL}$ vs $0.75 \pm 0.12, \mathrm{p} 0.008)$ that was associated $(|\mathrm{r}| 0.34, \mathrm{p}<0.0001)$ with haematocrit (coefficient $-0,01$, $\mathrm{t}$ value -3.87 ), and resting SBP $(-0.001,-2.18)$.

RBC NHE (5.6 $\pm 2.1 \mathrm{mmol} / \mathrm{L}$ h $v s 5.6 \pm 2.1)$, RBC vfcy $(12.4 \pm 3.7 \mu \mathrm{mol} / \mathrm{mL}$ h $v s 12.2 \pm 4.1)$, P SH $(408 \pm 62 \mu \mathrm{mol} / \mathrm{L} v s$ $423 \pm 60)$, RBC MDA ( $349 \pm 136 \mathrm{nmol} / \mathrm{L}$ vs $350 \pm 142)$, and $\mathrm{P}$ MDA (483 $\pm 224 \mathrm{nmol} / \mathrm{L} v s 498 \pm 257)$ did not differ between genders. 
Table 1. Clinical Characteristics, Demographic and Lifestyle Information of the Study Participants by Gender

\begin{tabular}{|c|c|c|c|}
\hline Age (years) & $46 \pm 13$ & $48 \pm 14$ & 0.4734 \\
\hline $\operatorname{BSA}\left(\mathrm{m}^{2}\right)$ & $1.69 \pm 0.16$ & $1.96 \pm 0.16$ & $<0.0001$ \\
\hline Never smoker/smoker/ex-smoker & $55 / 23 / 16$ & $37 / 18 / 20$ & 0.2922 \\
\hline Work (full-time/part-time/non-worker) & $43 / 18 / 33$ & $50 / 7 / 18$ & 0.0195 \\
\hline Type (sedentary/standing/manual/heavy) & $28 / 24 / 7 / 2$ & $25 / 14 / 9 / 9$ & 0.0511 \\
\hline Housework (h/day) & $2.8 \pm 1.3$ & $0.01 \pm 0.1$ & $<0.0001$ \\
\hline Physical activity (h/day) & $1.3 \pm 0.5$ & $1.7 \pm 0.6$ & $<0.0001$ \\
\hline
\end{tabular}

$\mathrm{BMI}=$ body mass index BSA $=$ body surface area .

Table 2. Biochemical Characteristics of the Study Participants by Gender

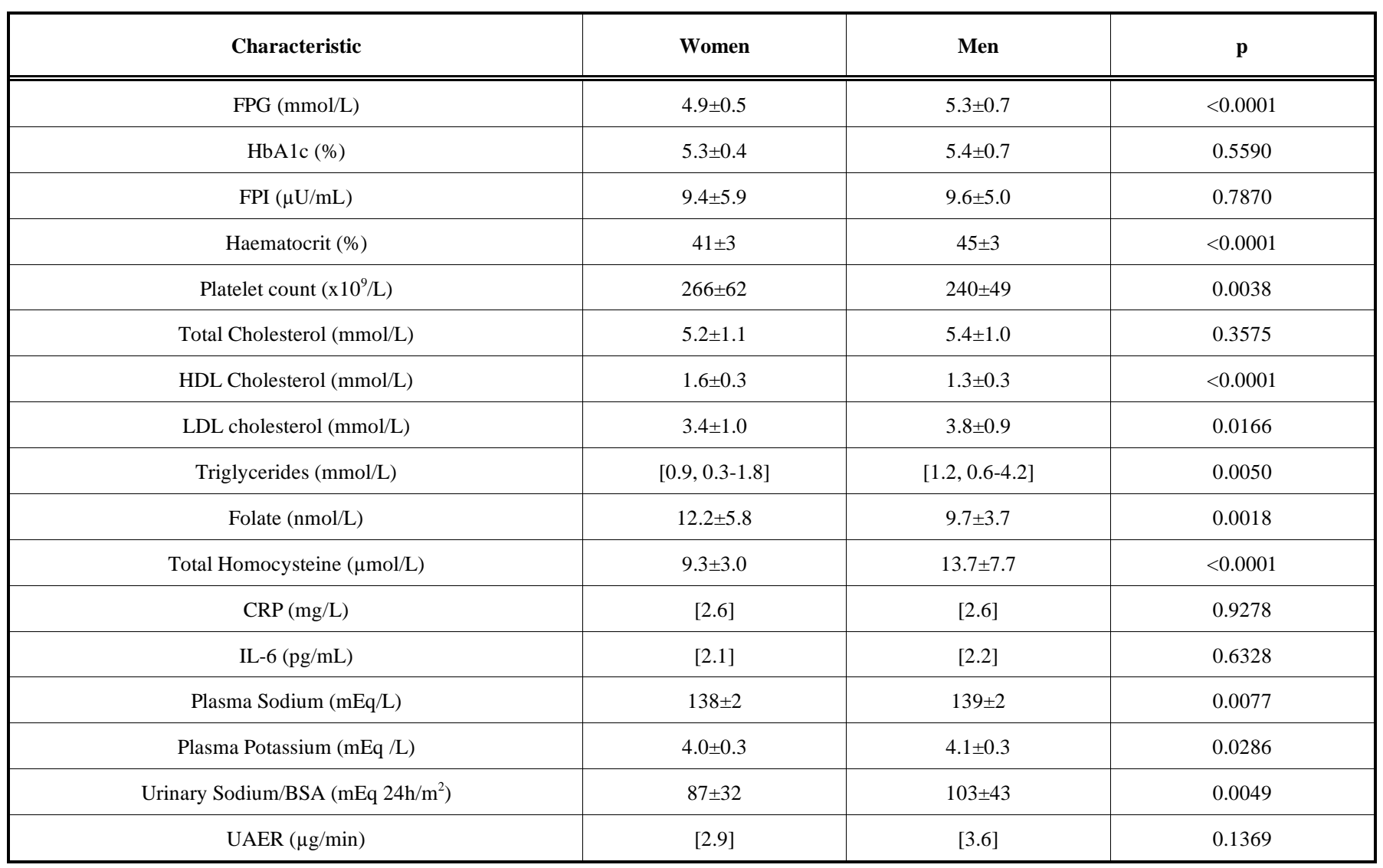

FPG = fasting plasma glucose; FPI = fasting plasma insulin; HDL = high density lipoprotein; LDL = low density lipoprotein; CRP = C-reactive protein; IL-6 = interleukin 6; BSA = body surface area; UAER = urinary albumin excretion rate. 


\section{Exercise Testing}

No patient presented basal ECG signs of left ventricular hypertrophy or exercise-induced angina. The maximum workload was significantly higher in men (Table 3). The major absolute increments in SBP and HR were observed in men (Fig. (1)), who generally reached their target HR and had a higher chronotropic index. Women, who had lower baseline levels of SBP and DBP than men, showed higher increments relative to maximum exercise capacity. Indeed, thirty-one (33\%) of women and $22(29 \%)$ of men had a HRE although female exercise capacity was significantly lower.

Subjects with a HRE were older $(53 \pm 13$ years vs $44 \pm 13$, $\mathrm{p}<0.0001)$ and had higher BMI $(27 \pm 4$ vs $25 \pm 4, \mathrm{p}<0.001)$, resting SBP $(134 \pm 15 \mathrm{mmHg}$ vs $117 \pm 15, \mathrm{p}<0.0001)$ and DBP $(86 \pm 10$ vs $76 \pm 10, \mathrm{p}<0.0001)$, plasma sodium $(139 \pm 2 \mathrm{mEq} / \mathrm{L}$ vs $138 \pm 2, \mathrm{p}<0.05)$, plasma total cholesterol $(5.6 \pm 1.0 \mathrm{mmol} / \mathrm{L}$ vs $5.2 \pm 1.0, \mathrm{p}<0.05)$, blood platelet counts $\left(270 \pm 68 \times 10^{9} / \mathrm{L} v s\right.$ $248 \pm 52, \mathrm{p}<0.05)$ and serum IL-6 $(2.8 \mathrm{pg} / \mathrm{mL}$ vs $2.3, \mathrm{p}<0.05)$.

Fifty-one (54\%) of women had a low chronotropic index, i.e. used up less than $80 \%$ of their HRR at the end of exercise, compared with $30(40 \%)$ of men (Fig. (1)).

Subjects with a low chronotropic index were more likely to be smokers $(33 \%$ vs $16 \%, \mathrm{p}<0.05)$ and had higher plasma fibrinogen $(8.1 \pm 1.7 \mu \mathrm{mol} / \mathrm{L} v s 7.8 \pm 1.4, \mathrm{p}<0.05)$, serum IL-6 (2.9 $\mathrm{pg} / \mathrm{mL}$ vs 2.0, p<0.0001), and RBC NHE activity $(5.9 \pm 2.3 \mathrm{mmol} / \mathrm{L} \mathrm{h}$ vs $5.2 \pm 1.8, \mathrm{p}<0.05)$. They achieved higher $\triangle \mathrm{ExSBP} / \mathrm{W} \quad(0.60 \pm 0.24$ vs $0.50 \pm 0.17, \mathrm{p}<0.01)$. Moreover, they spent more time in household chores $(1.8 \pm 1.8 \mathrm{~h} /$ day vs $1.3 \pm 1.5, \mathrm{p}<0.05)$ but less time in regular exercise $(1.8 \pm 1.4 \mathrm{~h} /$ week vs $2.7 \pm 2.1, \mathrm{p}<0.01)$.
HR recovery patterns after exercise did not differ between women and men. Nineteen women and 12 men had an abnormal HR recovery $(<22 \mathrm{bpm})$.

\section{Exploratory Data Analysis}

Baseline SBP was significantly associated ( $|\mathrm{r}| 0.62$, $\mathrm{p}<0.0001$ ) with age (coefficient 0.53 , $\mathrm{t}$ value 6.55), BSA (20.13, 3.86), HOMA IR $(15.68,3.66)$, plasma total cholesterol $(2.83,2.65)$, and plasma potassium $(-10.07,-2.65)$. Baseline DBP was only associated $(|\mathrm{r}| 0.52, \mathrm{p}<0.0001)$ with age $(0.24,4.61)$ and BMI $(0.97,5.31)$. Baseline HR correlated $(|\mathrm{r}| 0.37, \mathrm{p}<0.0001)$ with age $(-0.20,-2.35)$, haematocrit $(0.91,2.89)$, plasma folate $(19.24,3.29)$ and P MDA $(12.33$, 2.67).

$\triangle \mathrm{ExSBP} / \mathrm{W}$ correlated $(|\mathrm{r}| 0.55, \mathrm{p}<0.0001)$ with age (0.004, 3.81), BMI (0.01, 2.81), HbA1c (0.056, 2.02), time spent in housework $(0.026,3.02)$, time spent in cycling ($0.028,-3.06)$. In the multivariate analysis of covariance, remained predictive of the SBP increment both female gender (lambda 10.08, p 0.0018, power 0.903) and BMI (5.61, $0.0191,0.651)$; the covariate-factor interaction was significant (13.191, 0.0004, 0.967) (Fig. (2), upper panel).

$\Delta$ ExDBP/W correlated $(|\mathrm{r}| 0.40, \mathrm{p}<0.0001)$ positively with time spent in housework (coefficient 0.01 , t value 3.89), age $(0.001,2.36)$, and BMI $(0.003,2.19)$.

The chronotropic index correlated $(|\mathrm{r}| 0.40, \mathrm{p}<0.0001)$ with housework $(-0.040,-3.590)$, regular exercise $(0.020$, 2.358), BSA (-0.183, -2.115), and IL-6 (-0.086, -1.594). Having chronotropic incompetence was associated with cigarette use in the simple logistic regression model (likelihood

Table 3. Blood Pressure and Heart Rate Response to Exercise Testing of the Study Participants

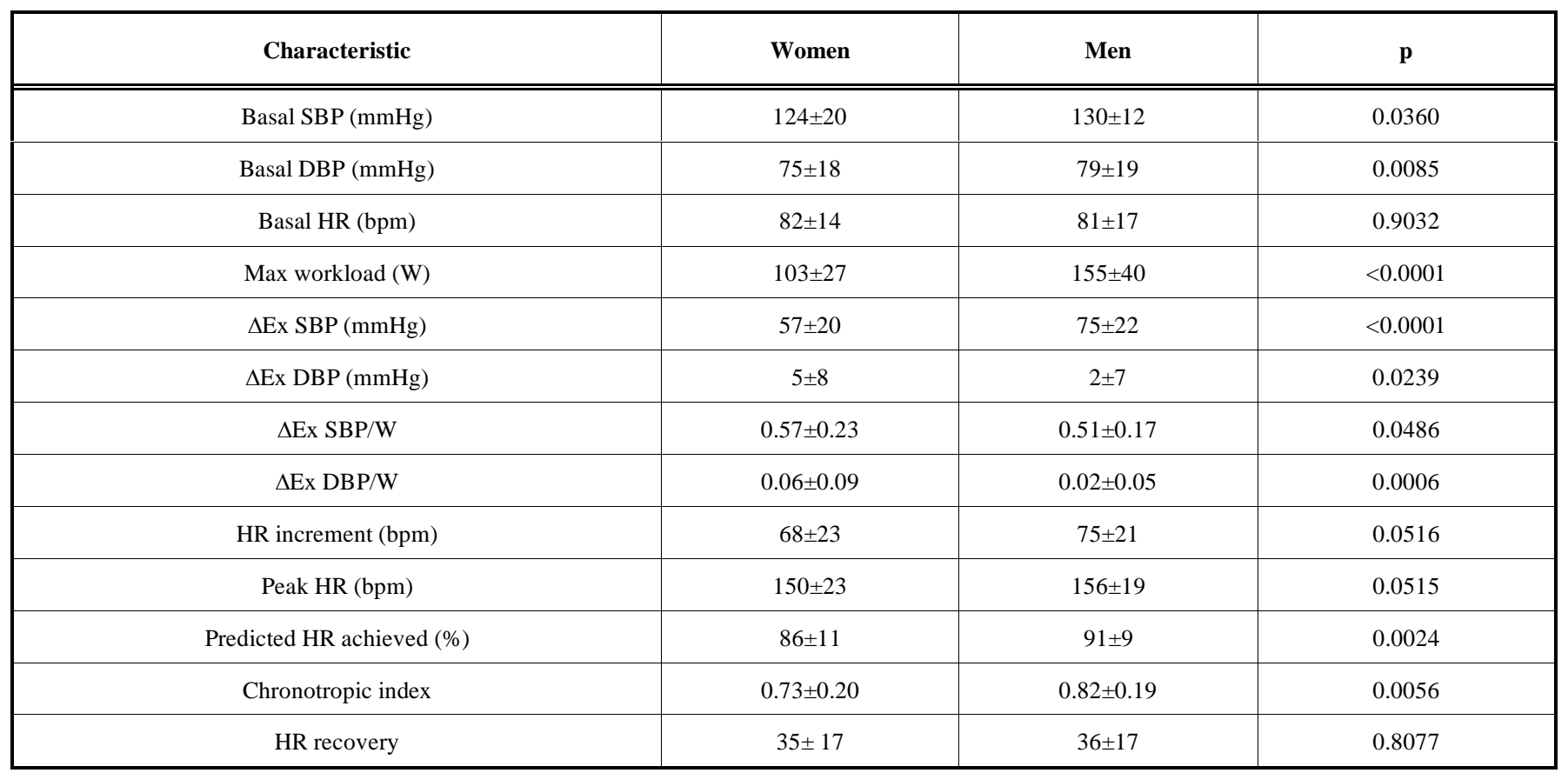

$\mathrm{SBP}=$ systolic blood pressure $; \mathrm{DBP}=$ diastolic blood pressure $; \mathrm{HR}=$ heart rate $\Delta \mathrm{Ex} \mathrm{SBP}=$ maximal exercise-induced change in $\mathrm{SBP} ; \Delta \mathrm{Ex} \mathrm{DBP}=$ maximal exercise-induced change in DBP; $\triangle \mathrm{Ex} \mathrm{SBP} / \mathrm{W}=$ maximal exercise-induced change in SBP relative to maximum exercise capacity; $\Delta \mathrm{Ex} \mathrm{DBP} / \mathrm{W}=$ maximal exercise-induced change in DBP relative to maximum exercise capacity. 


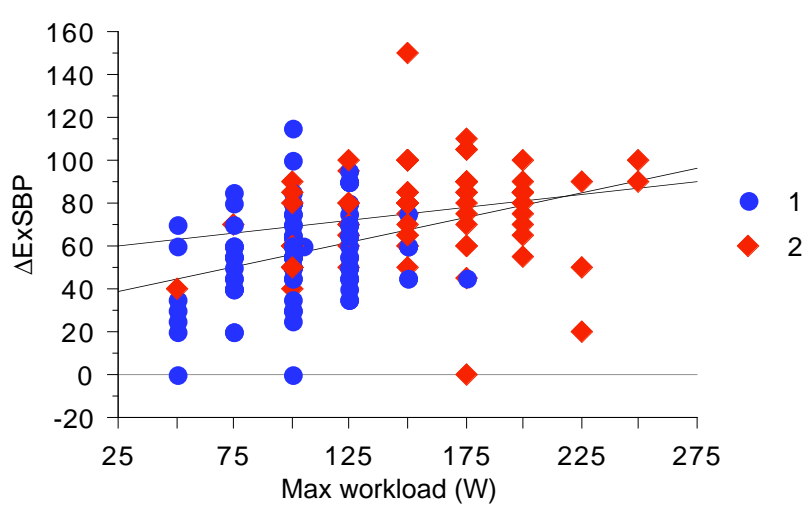

$\Delta \operatorname{ExSBP}=56,531+, 121 *$ Max workload $(\mathrm{W}) ; \mathrm{R}^{\wedge} 2=, 049(2)$

$\Delta \mathrm{ExSBP}=33,678+, 226 *$ Max workload $(\mathrm{W}) ; \mathrm{R}^{\wedge} 2=, 087(1)$

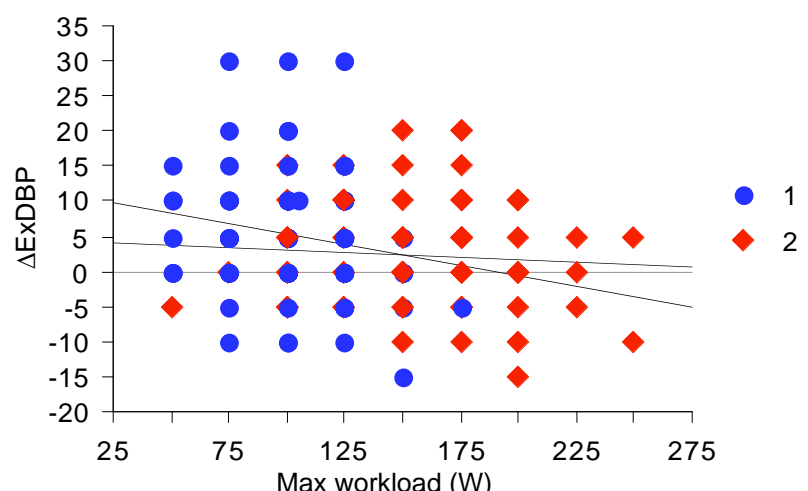

$\triangle \operatorname{ExDBP}=4,506-, 014{ }^{*}$ Max workload $(\mathrm{W}) ; \mathrm{R}^{\wedge} 2=, 006(2)$

$\triangle \operatorname{ExDBP}=11,325-, 06{ }^{*}$ Max workload $(\mathrm{W}) ; \mathrm{R}^{\wedge} 2=, 038(1)$

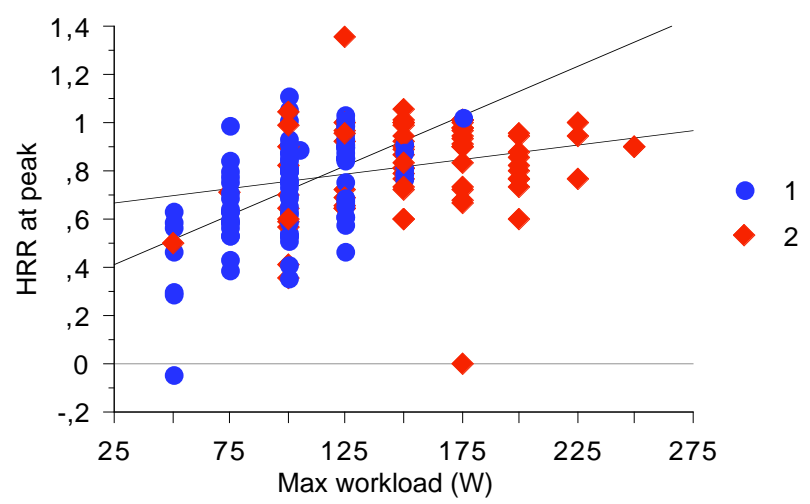

HRR at peak $=, 633+, 001{ }^{*}$ Max workload $(W) ; R^{\wedge} 2=, 062(2)$ HRR at peak $=, 311+, 004{ }^{*}$ Max workload $(W) ; R^{\wedge} 2=, 303(1)$

Fig. (1). Regression lines of the maximal increase in systolic blood pressure $(\triangle \mathrm{ExSBP})$, diastolic blood pressure $(\triangle \mathrm{ExDBP})$ and the fraction of heart rate response (HRR) $v s$ exercise workload (W) for women (1, blue circles) and men (2, red rhomboids). Respective regression equations are set below.

ratio test: $p=0.0076$, Chi-square 7.14$)$. In the multiple logistic regression (including gender, cigarette use, exercise, and IL-6), the relationship between smoking and low chronotropic index in men and women was explained away on the basis of serum concentration of IL-6 (likelihood ratio test: p value 0.0017, Chi-square 9.89) (Fig. (2), lower panel). Having chronotropic incompetence and/or abnormal heart rate recovery was associated with both serum concentrations of IL-6 (positively) and time engaged in regular exercise (negatively): the likelihood ratio test yielded a p of 0.0019 (Chi-square 9.69) and 0.014 (6.00), respectively (Fig. (3)).
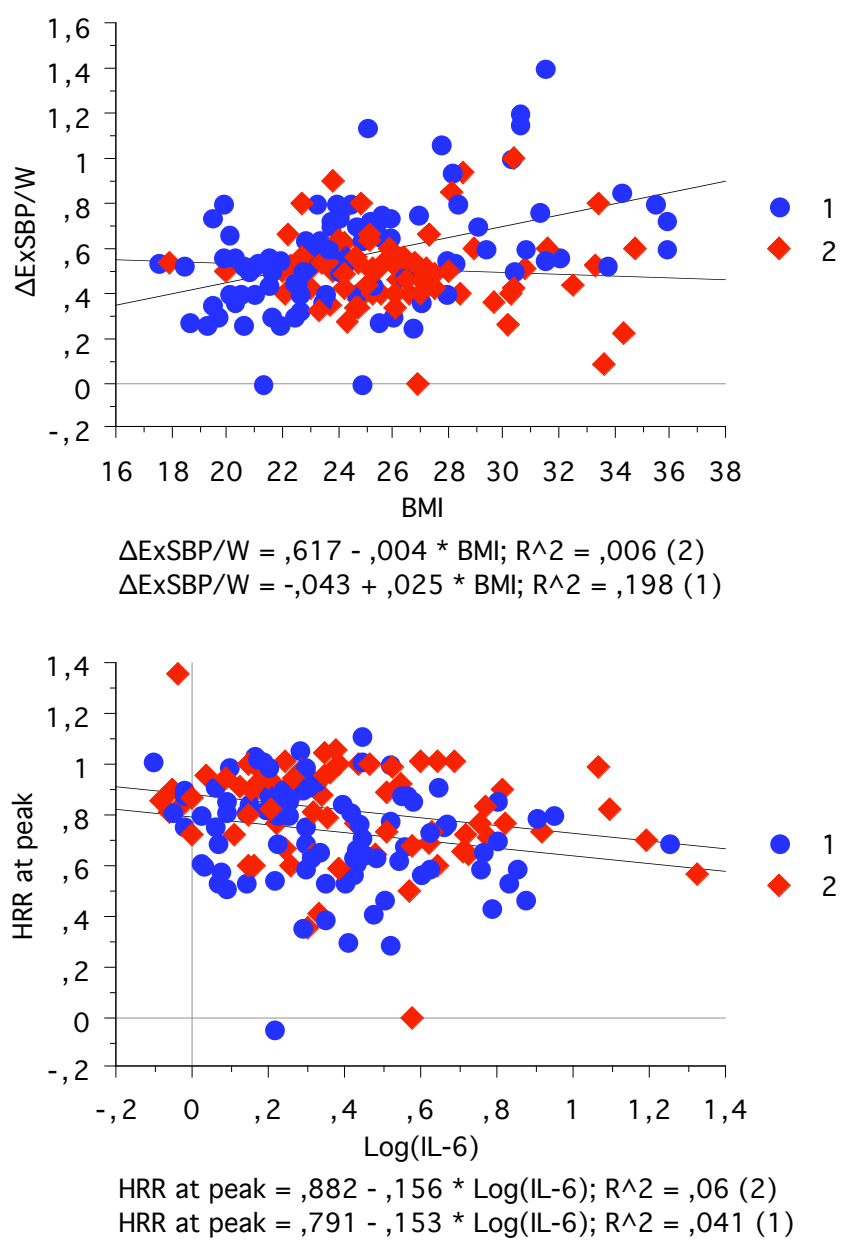

Fig. (2). In the upper panel, the relationship between BMI and the SBP increment relative to maximum exercise capacity ( $\triangle \mathrm{ExSBP} / \mathrm{W})$ for women (1, blue circles) and men (2, red rhomboids). In the lower panel, the relationship between chronotropic index (HRR at peak) and serum concentration of interleukin 6 in women (1, blue circles) and men (2, red rhomboids).

\section{DISCUSSION}

Using the Framingham Heart Study dataset, the effect of midlife cardiovascular risk factors on late-age survival has been estimated [12]. Physiological indices measured earlier in life can predict the residual life span distribution [13]. Thus, changing these respective indices could affect lifespan distributions, as observed recently in the Italian population. In this study, we evaluated several risk factors in conjunction with the haemodynamic responses during exercise in a group of healthy women and men. In addition to a proportion of smokers similar to that observed in men, our study identified a clustering of specific female cardiovascular risk factors: higher platelet counts, low participation in leisuretime aerobic physical activities, hypertensive response to exercise (BMI-dependent, unlike in men), and low fraction of heart rate reserve. Physical inactivity and high serum IL-6 levels (rather than cigarette use) were independently and 
significantly predictive of having both chronotropic incompetence and abnormal heart rate recovery.
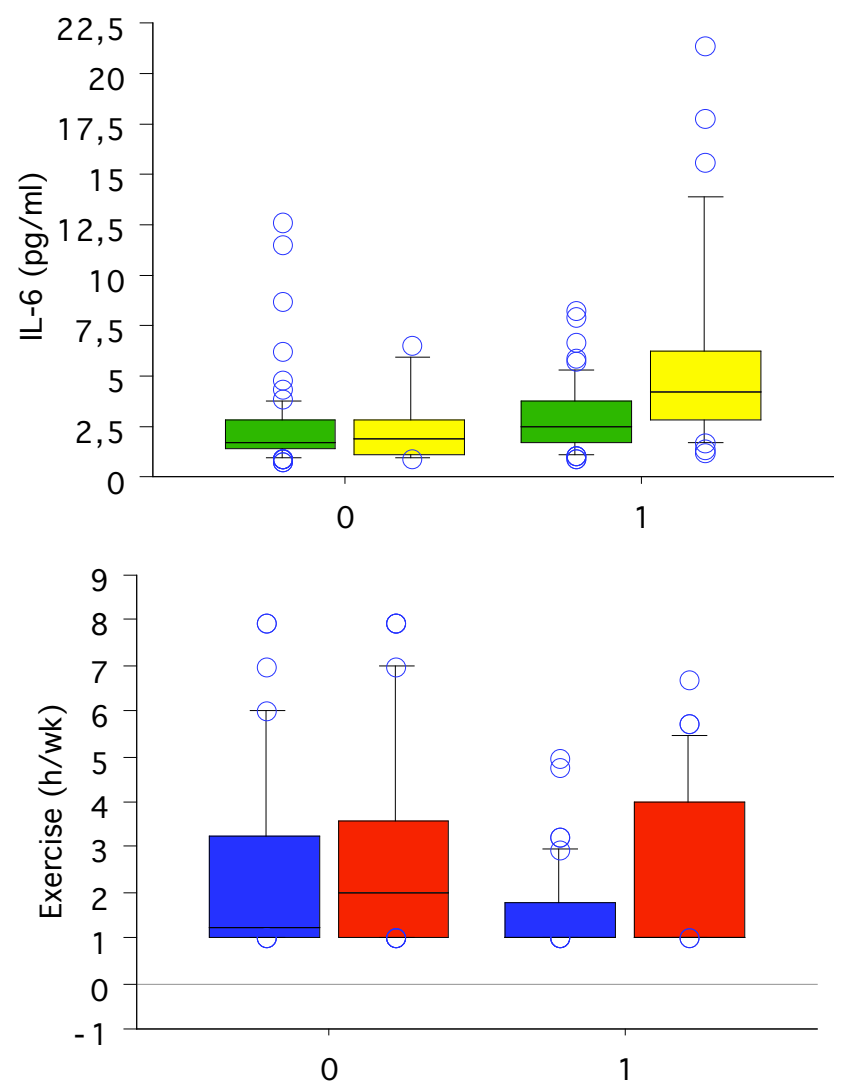

Fig. (3). In the upper panel, serum inteleukin 6 concentrations according to chronotropic index (normal in group 0 , or $<0.8$ in group 1 ) in non smokers (green boxes) and smokers (yellow boxes). In the lower panel, time spent in regular exercise (hours/week) according to chronotropic index (normal in group 0 , or $<0.8$ in group 1) in women (blue boxes) and men (red boxes).

At first glance, women showed a cardio-protective combination of clinical indices. Indeed, compared with men, they were leaner and had lower resting blood pressure. Genderassociated differences in blood pressure have been observed in humans and attributed to various potential mechanisms [14]. An increased salt dietary intake has been documented in our male sample.

Regarding demographic characteristics, women reported similar smoking prevalence than men and significantly lower participation in standard aerobic physical activities, with the exception of household chores that seemed to be an exclusive prerogative of female gender in Italy. The American Heart Association classified physical inactivity as a major cardiovascular risk factor [15]. In a population of Dutch women who participated in the EPIC in 1993-1995, blood pressure was most clearly associated with time spent in sports. Leisure-time activity was inversely related to cardiovascular disease risk indicators, but work activity and housework were not [16]. Italian women reported low levels of regular participation in sports. Educational levels were associated with lower household and higher leisure-time physical activity; in turn, regular physical activity influenced physical work capacity at the bicycle ergometer.
Additional peculiar female haematological features were the lower level of haematocrit and the higher platelet counts. Sample size did not allow a close evaluation of the relation between female hormonal state and platelet count. Differences in platelet counts by sex have been reported [17]. Mild platelet count elevation is a risk factor for venous thrombosis; its contribution to the rates of cardiovascular events is still to be explored despite its possible correlation with inflammatory markers and long-term mortality in unstable angina [18].

From the biochemical viewpoint, female gender seemed favoured by nature. In women, blood levels of glucose, triglycerides, and homocysteine were lower, whereas those of HDL-cholesterol and folate were higher. RBC GSH, i.e. cellular antioxidant protection, was heightened. It has been consistently reported that women have lower FPG levels than men and display a more rapid fall in plasma glucose during prolonged fasting [19]. FPG has been found independently associated with resting and exercise blood pressures and development of hypertension after 7-years followup in healthy men [20]. Interestingly, in our study, insulin resistance by HOMA ${ }_{\text {IR }}$ was significantly associated not only with body mass, as expected, but also with a marker of oxidative damage (P MDA). In turn, resting systolic blood pressure increased with increasing age, BSA, HOMA ${ }_{I R}$, plasma total cholesterol, whereas it showed an inverse relationship with plasma potassium. Sex differences in sodium, potassium, and water regulation have been suggested involved in the lowering of cardiovascular disease risk among women [21, 22]. Resting diastolic blood pressure was positively related with age and body mass. The resting heart rate declined with age, while it showed a positive correlation with haematocrit, plasma folate, and P MDA. Heart rate positive correlation with folate deserves attention since pulse rate has been found to decline both with ageing [12] and regular exercise. Exercise has been implicated in the up-regulation of neuronal nitric oxide synthase, which results in vagal bradycardia in vitro and in animals. The molecular mechanisms responsible may involve interplay between haemodynamic elements and oxidative stress [23]. Increased radical production ( $\mathrm{P}$ MDA) could stimulate nitric oxide formation that critically depends on the availability of folic acid.

Oxidative stress is thought to be involved in ageassociated diseases. GSH content decreases with age in many tissues, whereas it is unclear whether it differs between genders. So far, no gender specific difference in RBC GSH content was found, although 1) estrogen is one of the hormones that regulate GSH homeostasis, and 2) age- and-gender dependent alterations in the activities of glutathione-related enzymes have been observed [24, 25]. Our finding of a gender-dependency in RBC GSH content is partially at variance with previous data in mice where no difference was found. The result could depend on the human population studied or on the analytical procedure that was performed immediately after blood withdrawal without prolonged sample manipulation.

Exercise ECG is the most commonly used form of stress testing, frequently evaluated in women even though ECG changes during exercise are less sensitive and specific in women as compared with men [26]. Gender differences exist 
in the ventilatory response to progressive exercise [27] and the progression of metabolic responses [28]. Contrary to the other component of cardiac power output, however, mean arterial pressure has been reported to be independent of body size [29].

Examined women had lower baseline blood pressure than men. Nonetheless, their response to exercise was characterised by major increases in maximal SBP and DBP relative to maximal achieved workloads. The enhanced SBP response to graded exercise was associated positively with age, BMI, $\mathrm{HbA1c}$, and time spent in household chores, while negatively with regular cycling. The enhanced DBP response was influenced by time dedicated to housework more than by age and body mass. A HRE has been reported to predict the future development of hypertension, whereas its prognostic significance with regard to likelihood of myocardial perfusion abnormalities and mortality rate is unclear [8]. It has been associated with a high prevalence of transient ischaemic dilation of the left ventricle in patients without other significant perfusion defects [30]. Intriguingly, only women were particularly susceptible to the potentiating effects of body mass on the SBP response to exercise. An abnormal blood pressure response was also associated with $\mathrm{HbA} 1 \mathrm{c}$ levels and lack of physical fitness (more time spent in housework than in exercise). Indeed, protein glycosylation may affect artery compliance in healthy subjects [7].

Chronotropic incompetence is predictive of increased allcause mortality and coronary heart disease incidence; moreover, considering both chronotropic incompetence and abnormal heart rate recovery most powerfully stratified risk [31]. Physical inactivity and high serum IL-6 levels were independently predictive of having both these pathological responses. Compared with men, a large proportion of women showed an impaired HR reserve. Chronotropic incompetence was more frequent in smokers who had abnormal inflammatory (IL-6 and fibrinogen) and oxidative (RBC NHE) biomarkers. IL-6 belongs to the class of systemic inflammatory markers; its circulating levels have been associated with coronary artery disease risk [32].

In summary, our study identified a set of gender-specific cardiovascular risk factors potentially capable of predicting survival characteristics, which could be used as indicators for health surveillance programmes in Italy (and elsewhere). An analysis of longitudinal data containing regular measurements of these physiological indices in numerous individuals could help us understand their predictive ability.

\section{ACKNOWLEDGEMENTS}

We are obliged to Dr Javie Rosada and Marco Pinelli for their help with exercise testing and to Prof. G. Mariani for insulin assay.

\section{REFERENCES}

[1] Bobak M. Relative and absolute gender gap in all-cause mortality in Europe and the contribution of smoking. Eur J Epidemiol 2003; 18: $15-8$.

[2] Kesteloot H, Sans S, Kromhout D. Dynamics of cardiovascular and all-cause mortality in Western and Eastern Europe between 1970 and 2000. Eur Heart J 2006; 27: 107-13.

[3] Matteucci E, Giampietro O. Oxidative stress in families of type 1 diabetic patients. Diabetes Care 2000; 23: 1182-6.
[4] Matteucci E, Giampietro O. Oxidative stress in families of type 1 diabetic patients: further evidence. Diabetes Care 2001; 24: 167-8.

[5] Matteucci E, Giampietro O. Transmembrane electron transfer in diabetic nephropathy. Diabetes Care 2000; 23: 994-9.

[6] Matteucci E, Passerai S, Mariotti M, et al. Dietary habits and nutritional biomarkers in italian type 1 diabetes families. Evidence of unhealthy diet and combined-vitamin deficient intakes. Eur J Clin Nutr 2005; 59: 114-22.

[7] Matteucci E, Rosada J, Pinelli M, Giusti C, Giampietro O. Systolic blood pressure response to exercise in type 1 diabetes families compared with healthy control individuals. J Hypertens 2006; 24: 1745-51.

[8] Campbell L, Marwick TH, Pashkow FJ, Snader CE, Lauer MS. Usefulness of an exaggerated systolic blood pressure response to exercise in predicting myocardial perfusion defects in known or suspected coronary artery disease. Am J Cardiol 1999; 84: 130410

[9] Okin PM, Lauer MS, Kligfield P. Chronotropic response to exercise. Circulation 1996; 94: 3226-31.

[10] Myers J, Tan SY, Abella J, Aleti V, Froelicher VF. Comparison of chronotropic response to exercise and heart rate recovery in predicting cardiovascular mortality. Eur J Cardiovasc Prev Rehabil 2007; 14: 215-21.

[11] Matteucci E, Malvaldi G, Fagnani F, Evangelista I, Giampietro O. Redox status and immune function in type 1 diabetes families. Clin Exp Immunol 2004; 136: 549-54.

[12] Terry DF, Pencina MJ, Vasan RS, et al. Cardiovascular risk factors predictive for survival and morbidity-free survival in the oldest-old Framingham Heart Study participants. J Am Geriatr Soc 2005; 53: 1944-50.

[13] Yashin AI, Akushevich IV, Arbeev KG, Akushevich L, Ukraintseva SV, Kulminski A. Insights on aging and exceptional longevity from longitudinal data: novel findings from the Framingham Heart Study. Age Dordr Neth 2006; 28; 363-74.

[14] Reckelhoff JF. Gender differences in the regulation of blood pressure. Hypertension 2001; 37: 1199-208.

[15] Grundy SM, Pasternak R, Greenland P, Smith S, Fuster V. AHA/ACC scientific statement: Assessment of cardiovascular risk by use of multiple-risk-factor assessment equations. A statement for healthcare professionals from the American Heart Association and the American College of Cardiology. J Am Coll Cardiol 1999; 34: $1348-59$.

[16] Pols MA, Peeters PH, Twisk JW, Kemper HC, Grobbee DE. Physical activity and cardiovascular risk profile in women. Am J Epidemiol 1997; 146: 322-8.

[17] Segal JB, Moliterno AR. Platelet counts differ by sex, ethnicity, and age in the United States. Ann Epidemiol 2006; 16: 123-30.

[18] Mueller C, Neumann FJ, Hochholzer W, et al. The impact of platelet count on mortality in unstable angina/non-ST-segment elevation myocardial infarction. Am Heart J 2006; 151: 1214.e1-7.

[19] Pomerleau J, McKeigue PM, Chaturvedi N. Relationships of fasting and postload glucose levels to sex and alcohol consumption. Are American Diabetes Association criteria biased against detection of diabetes in women? Diabetes Care 1999; 22: 430-3.

[20] Biornholt J, Erikssen G, Kjeldsen SE, Bodegård J, Thaulow E, Erikssen J. Fasting blood glucose is independently associated with resting and exercise blood pressure and development of elevated blood pressure. J Hypertens 2003; 21: 1383-9.

[21] Stachenfeld NS, Splenser AE, Calzone WL, Taylor MP, Keefe DL. Sex differences in osmotic regulation of AVP and renal sodium handling. J Appl Physiol 2001; 91: 1893-901.

[22] Zheng W, Shi M, Yoo S-E, Ji H, Roesch DM. Estrogens contribute to a sex difference in plasma potassium concentration: a mechanism for regulation of adrenal angiotensin receptors. Gend Med 2006; 3: 43-53.

[23] Danson EJ, Mankia KS, Golding S, et al. Impaired regulation of neuronal nitric oxide synthase and heart rate during exercise in mice lacking one nNOS allele. J Physiol 2004; 558: 963-74.

[24] Wang H, Liu H, Liu R-M. Gender difference in glutathione metabolism during aging in mice. Exp Gerontol 2003; 38: 507-17.

[25] Habif S, Mutaf I, Turgan N, et al. Age and gender dependent alterations in the activities of glutathione related enzymes in healthy subjects. Clin Biochem 2001; 34: 667-71.

[26] Mieres JH, Shaw LJ, Arai A, et al. Cardiac Imaging Committee, Council on Clinical Cardiology, and the Cardiovascular Imaging and Intervention Committee, Council on Cardiovascular Radiology 
and Intervention, American Heart Association. Role of noninvasive testing in the clinical evaluation of women with suspected coronary artery disease: Consensus statement from the Cardiac Imaging Committee, Council on Clinical Cardiology, and the Cardiovascular Imaging and Intervention Committee, Council on Cardiovascular Radiology and Intervention, American Heart Association. Circulation 2005; 111: 682-96.

[27] Kilbride E, McLoughlin P, Gallagher CG, Harty HR. Do gender differences exist in the ventilatory response to progressive exercise in males and females of average fitness. Eur J Appl Physiol 2003; 89: 595-602.

[28] Kang J, Hoffman JR, Chaloupka EC, Ratamess NA, Weiser PC. Gender differences in the progression of metabolic responses during incremental exercise. J Sports Med Phys Fitness 2006; 46: 718 .
[29] Döbeln WV. Maximal oxygen intake, body size, and total haemoglobin in normal men. Acta Physiol Scand 1956; 38: 193-9.

[30] Smelley MP, Virnich DE, Williams KA, Ward RP. A hypertensive response to exercise is associated with transient ischaemic dilation on myocardial perfusion SPECT imaging. J Nucl Cardiol 2007; 14: 537-43.

[31] Lauer MS, Okin PM, Larson MG, Evans JC, Levy D. Impaired heart rate response to graded exercise. Prognostic implications of chronotropic incompetence in the Framingham Heart Study. Circulation 1996; 93: 1520-6.

[32] Danesh J, Kaptoge S, Mann AG, et al. Long-term interleukin-6 levels and subsequent risk of coronary heart disease: two new prospective studies and systematic review. PloS Med 2008; 5: e78.

(C) Matteucci and Giampietro et al.; Licensee Bentham Open.

This is an open access article licensed under the terms of the Creative Commons Attribution Non-Commercial License (http://creativecommons.org/licenses/by-nc/3.0/) which permits unrestricted, non-commercial use, distribution and reproduction in any medium, provided the work is properly cited. 